\title{
A MULTIGRID-BASED BEAM DYNAMICS CODE FOR HIGH CURRENT PROTON LINACS
}

\author{
Paolo Pierini ${ }^{\dagger}$, Giovanni Bellomo and Carlo Pagani, \\ INFN Milano LASA, Via Fratelli Cervi, 201 I-20090 Segrate (MI) Italy
}

\begin{abstract}
A beam dynamics code has been developed for the design assessment of a superconducting high current proton linac for the ENEA/INFN TRASCO Project (intended for nuclear waste transmutation in a subcritical reactor). The code deals with the dynamics of a moderate energy (above tens of $\mathrm{MeV}$ ) and high current proton beam in a beamline composed of elliptical superconducting cavities and quadrupoles. A 3D Poisson solver based on multigrid techniques has been chosen for the space charge calculations. The simulation results for the reference linac design, with a particular care to emittance growth and halo generation, are here reported.
\end{abstract}

\section{GENERAL FEATURES OF THE CODE}

The code[1] has been specifically developed for the simulation of high current proton beams in a superconducting linac that uses "elliptical" cavities, as is the case for TRASCO[2]. The beam is advanced along the linac in phase steps (where the phase is defined as $\phi=\omega_{r f} t$, and $\omega_{r f}$ is $2 \pi$ the frequency of the linac). In case a particle crosses an element boundary during a timestep, a substep is performed up to the element end and the step is then continued in the following element. Particles crossing an element boundary where output is desired are collected in buffers for disk storage.

The beamline is composed of a sequence of elements of different types: quadrupoles, drift spaces and cavities. An "ideal" 3D uniform focussing element can also be used for analytical checks of the code.

For the propagation in the quadrupoles and drifts analytical maps are used (which include chromatic effects for the quadrupole), whereas for the RF cavities a direct integration in the cavity field is performed. A cavity is described either with an analytical field shape of an "ideal" reduced $\beta$ cavity or by an on-axis field map (provided by any cavity electromagnetic code). Off-axis expansion, to second order, of the electric and magnetic fields is computed for particle tracking.

Space charge is computed in multiples of the particle tracking phase steps. The space charge kicks can be evaluated either using a direct point to point method or with a fast 3D Poisson solver in the reference particle rest frame, based on a multigrid algorithm.

\subsection{RF Cavities Modeling}

The codes used for the design of the TRASCO linac replace the elliptical RF cavities with a properly chosen "ideal" cavity with an analytical field given by:

$E_{z}(z, t)=2 E_{a c c} \sin \left(\omega_{r f} t+\varphi_{c}\right) \sin \left(\frac{\omega_{r f} z}{c \beta_{c}}\right)$

where $E_{a c c}$ is the accelerating field, $\beta_{c}$ the cavity synchronous velocity and $\varphi_{c}$ the phase. Both $E_{a c c}$ and $\beta_{c}$ are defined so that the cavity gives the same energy gain of the "real" cavity (as designed by a program as Superfish[3]) in all the energy range of the linac where the cavity is used. Note that $\beta_{c}$ does not depend from the inner cell length, it rather comes from a "dynamical" equivalence of a real cavity with an ideal sine-like cavity.

This equivalence has been tested against a much simpler (although much quicker in terms of computational costs) model of a cavity: the RF gap. The transfer matrix elements for the longitudinal phase space $(\Delta \phi, \Delta E)$ are plotted in Figure 1 for three cavity models: the direct integration in the $E_{z}$ field, the sine-like approximation defined above and the RF gap equivalence, where the whole 5 cell cavity is replaced by a single RF gap, giving the right energy gain and phase advance at each energy. The plots were computed for the $\beta$ range corresponding to the first section of the TRASCO linac (100-190 MeV).
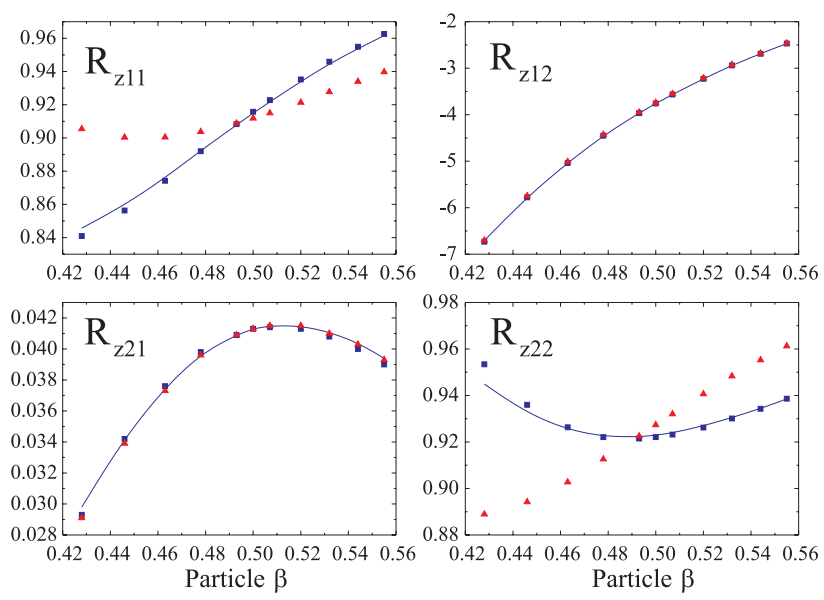

Figure 1: Longitudinal transfer matrix elements for the three cavity models: direct integration (solid line), sinelike equivalent (squares, see text) and RF gap (triangles). Phase space units are deg and MeV. $\varphi_{s}=-30 \mathrm{deg}$.

† Email: Paolo.Pierini@mi.infn.it 
The transfer matrix of the transverse plane $(x, d x / d s)$ is shown in Figure 2, for the same cavity models of Figure 1.

It is worth noting that the largest errors are found on the elements on the main diagonal of the transverse and longitudinal transfer matrices, where the sine-like equivalent has an error of $10^{-3}-10^{-4}$, whereas the RF gap gives a difference of the order of $10^{-2}$.

In both the transverse and longitudinal matrix cases, when the cavity is operated near its nominal velocity (here $\beta=0.5$ ) the three models give approximately the same values for the elements (to few parts in 10,000).
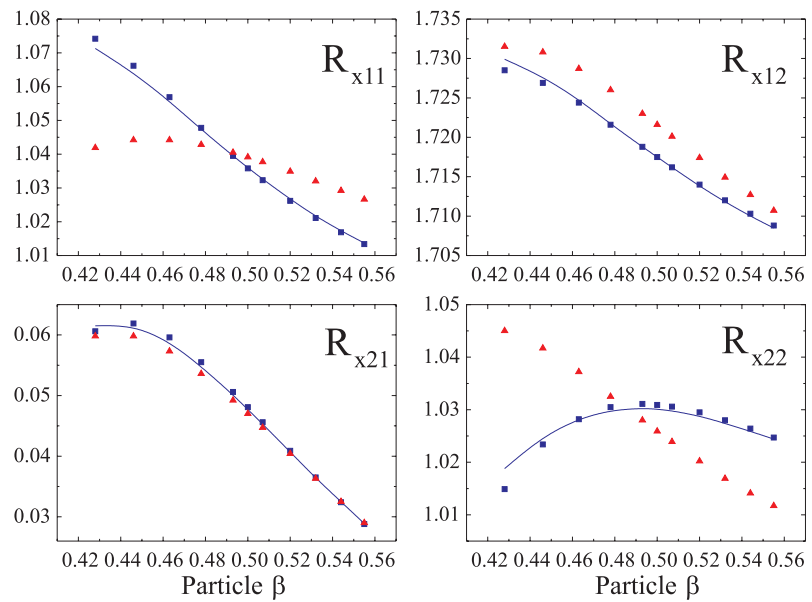

Figure 2: Transverse transfer matrix elements for the three cavity models: direct integration (solid line), sine-like equivalent (squares, see text) and RF gap (triangles). Phase space units are $\mathrm{mm}$ and $\operatorname{mrad} . \varphi_{s}=-30 \mathrm{deg}$.

\subsection{The Space Charge Multigrid Algorithm}

Since the code advances in time (phase), when the space charge calculation is needed the particle coordinates are Lorentz-transformed to the rest frame of the reference particle, the charge distribution is evaluated using a cloud in cell (CIC) assignment scheme and the 3D Poisson equation is solved.

The fields computed from the solution potential are then transformed back in the laboratory frame and the force is interpolated on the particle position using trilinear interpolation[4].

The Poisson equation is solved iteratively using a multigrid solver[5], a fast iterative method that uses standard relaxation algorithms (in our case we chose the Gauss-Seidel) and combines it with the discretization of the problem on a series of nested grid levels (in our case obtained by step doubling of the finest level).

The code implements both the V-cycle and the full multigrid[5] cycling strategies, but the V-cycle allows to make use of the potential map computed at a previous space charge computation in order to start from a better estimate for the solution potential. This strategy leads to a speed up of the space charge calculations (reducing the time by a typical factor of $30 \%$ ), in order to achieve the specified convergence[1].
The restriction operator (needed to transfer quantities to a coarser mesh level) is the trilinear averaging of the 27 neighboring nodes and the prolongation operator (needed to transfer the solution to a finer mesh level) is the trilinear interpolation.

The space charge routine based on the multigrid solver has been thoroughly tested, both with a comparison with the point to point space charge calculation (which, however, is limited to a small number of interacting particles, due to the time scaling with the squared number of particles) and with simple analytical cases. A report of the tests can be found in Reference 1 .

Typical TRASCO simulations are performed with $10^{4}$ $10^{5}$ particles, a phase step of a few (2-4) RF degrees, a space charge step every 5-15 phase steps using a mesh of $33 \times 33 \times 33$ points, extending to $\pm 5-6 \mathrm{rms}$ beam sizes. The whole linac runs in a few hours on a desktop system.

\section{THE TRASCO LINAC SIMULATIONS}

The $352 \mathrm{MHz}$ superconducting linac accelerates a $25 \mathrm{~mA}$ proton beam from $100 \mathrm{MeV}$ to a maximum energy of $1.6 \mathrm{GeV}$. The linac is divided in three sections, with five cell cavities designed for a synchronous $\beta$ of $0.5,0.65$ and 0.85 , grouped in cryostats containing 2,3 and 4 structures each. The focussing is provided by a periodic array of quadrupole doublets between the cryostats and the lattice cells are 8.03, 11.152 and 15.34 meters each.

Beam dynamics studies of the TRASCO linac using the code described here, with a number of simulation particles ranging from few thousands to $10^{5}$, has been already reported in Refs. 6 and 7.

In Figure 3 we present the rms and $90 \%$ beam normalized emittances along the linac. The rms emittance growth is less than $4 \%$ in all planes. The beam distribution is well matched along the linac, as shown by the nearly constant behavior of the $90 \%$ emittance curves. No sign of particle drifting away from the beam core can be seen from the phase space plots.

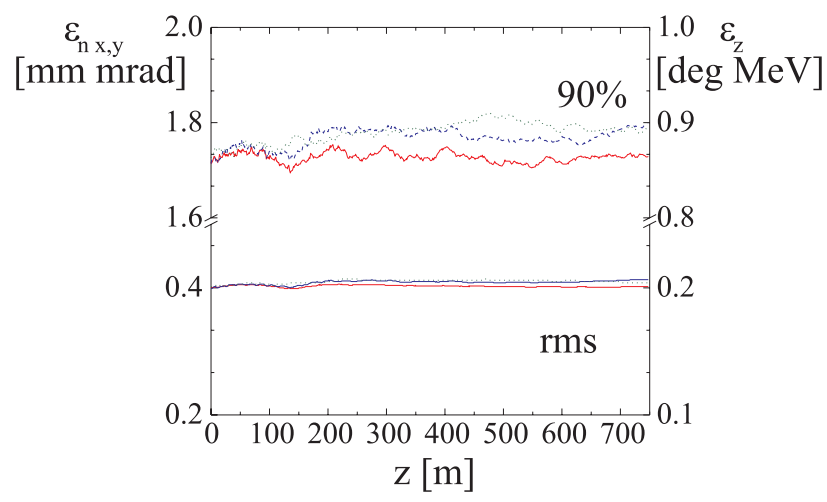

Figure 3: Normalized emittances along the linac. Bottom lines rms values, upper lines $90 \%$ beam emittance. The $\mathrm{x}$ plane is the solid line, the y plane is the dashed line and the $\mathrm{z}$ plane (on the right axis) is the dotted line. The simulation was performed with 10,000 particles. 


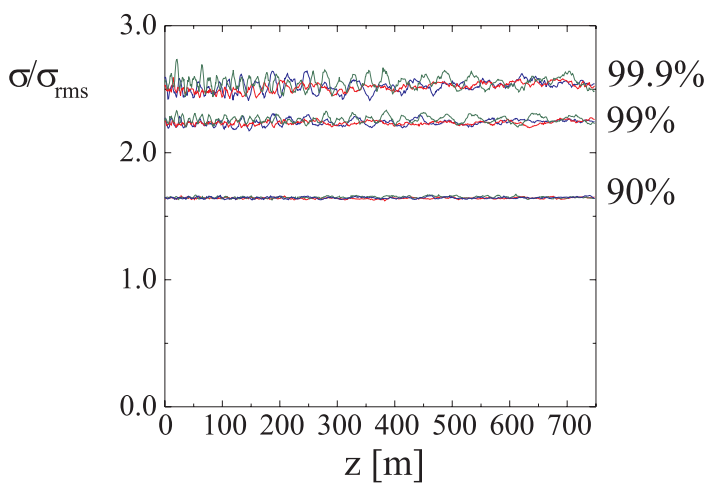

Figure 4: Ratio of the sizes containing different particle populations (including, from bottom to top $90 \%, 99 \%$ and $99.9 \%$ of the beam, respectively) to the rms beam size.

Another sign of the absence of beam tail formation can be seen from Figure 4, where we plot the ratio of the size containing $90 \%, 99 \%$ and $99.9 \%$ of the beam to the rms beam size. This beam size fraction is approximately constant (averaging the numerical noise due to the poor statistics on the higher values of beam size fractions) along the linac, and confirms that particles are not drifting to the tails of the distribution, as would be the case for a charge redistribution process, that could give rise to a large beam halo.

The matched beam conditions at the linac input and the matching between the three focussing sections of the linac have been determined with the linear dynamics code, TRACE3-D[8]. The matching between the sections is obtained using the gradients of two quadrupole doublets and the synchronous phase of the cavities in two cryomodules at the section interface. RF cavities are modeled in TRACE3-D with a modified tank element (type 13), consistently with the multiparticle simulations.

The phase advances per cell are always lower than 90 deg in all linac sections. The phase advances per meter, as computed by the beam dynamics code from the rms beam parameters, can be seen in Figure 5.

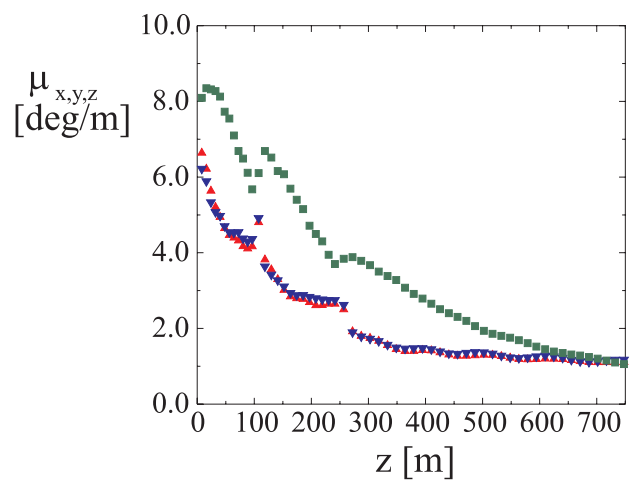

Figure 5: Phase advances per meter (up triangles: $\mathrm{x}$, down triangles: $y$, squares: $\mathrm{z}$ ) along the linac, as computed from the rms beam during the simulation. The cell lengths of the three sections are $8.03 \mathrm{~m}, 11.152 \mathrm{~m}$ and $15.34 \mathrm{~m}$, respectively.
The rms beam envelopes plotted in Figure 6 show a discontinuity of the beam sizes at the interfaces, and a small transverse mismatch in the third linac section (visible also as oscillations in Figure 5 and due to the approximations of the TRACE3-D matching). However, no evident emittance growth is produced by these discontinuities and by the beam mismatch.

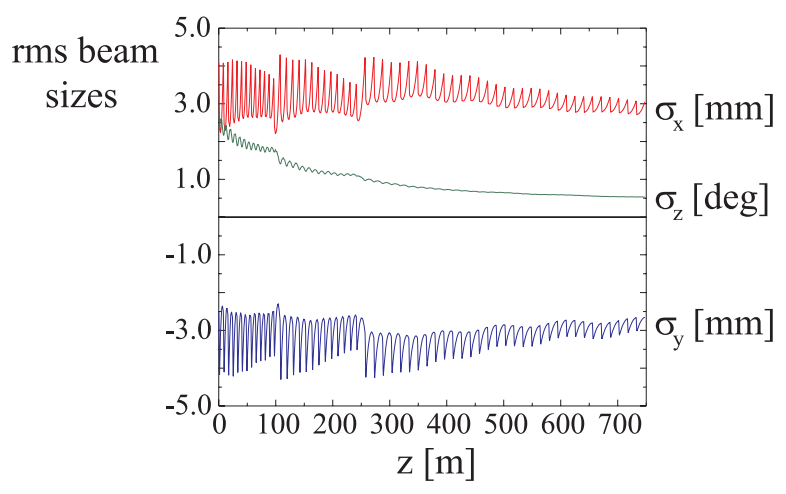

Figure 6: rms beam sizes in the TRASCO linac.

\section{CONCLUSIONS}

A multiparticle space charge code has been developed for high current superconducting proton linacs with elliptical cavities. The code uses an accurate cavity modeling, that reproduces the cavity behavior in the large velocity region in which the cavities are effectively used. Space charge is included with a 3D iterative Poisson solver, based on multigrid methods.

The code has been successfully used since two years to assess the design of the TRASCO linac.

\section{ACKNOWLEDGEMENTS}

We thank N. Pichoff (CEA/Saclay) for the many discussions and his help in the multigrid algorithm testing.

\section{REFERENCES}

[1] P. Pierini, "A Multigrid-Based Approach to Modeling a High Current Superconducting Linac for Waste Transmutation", in Proceedings of the 1998 Int. Computational Acc. Physics Conf., September 14-18 1998, Monterey, CA.

[2] C. Pagani, D. Barni, G. Bellomo, R. Parodi, P. Pierini, "Status of the INFN High Current Proton Linac for Nuclear Waste Transmutation", in Proceedings of the XIX International Linear Accelerator Conference, August 23-28 1998, Chicago, IL.

[3] J.H. Billen and L.M. Young, "Poisson Superfish", LA-UR-961834, Los Alamos, NM, revised 1999.

[4] "Computer Simulations Using Particles", by R.W. Hockney and J.W. Eastwood, Adam Hilger, New York, NY 1988, ISBN 085274-392-0.

[5] "A Multigrid Tutorial", by William Briggs, SIAM, Philadelphia, PA 1987, ISBN 0-89871-222-1.

[6] G. Bellomo, P. Pierini, "Beam Dynamics In A Proposed $350 \mathrm{MHz}$ SC Linac For Nuclear Waste Transmutation And Energy Production", in Proceedings of the 1998 European Particle Accelerator Conference, Stockholm, June 21-26 1998.

[7] G. Bellomo, P. Pierini, "Beam Dynamics in a High Current SC Proton Linac for Nuclear Waste Transmutation", in Proceedings of the XIX International Linear Accelerator Conference, August 23-28 1998, Chicago, IL.

[8] K. Krandall, D. Rushtoi, "TRACE3-D Documentation", LA-UR97-886, Los Alamos, NM, 1987. 